Z. Klin. Chem. Klin. Biochem.

10. Jg. 1972 , S. $450-452$

\title{
Nachweis von Proteaseninhibitoren im Intestinaltrakt von Mensch und Hund
}

\author{
Von K. Hochstrasser, G. Bickel, R. Reichert, H. Feuth und D. Mé́ki \\ Biochemisches Laboratorium (Leitung: PD Dr. K. Hochstraßer) der HNO-Klinik (Direktor: Prof. Dr. H. H. Naumann) \\ der.Universität München
}

(Eingegangen am 7. August/3. Oktober 1972)

Herrn Prof. Dr. Dr. E. Werle zum 7o. Geburtstag

Aus Homogenaten von menschlichem Colon sowie Duodenum, Jejunum, Ileum und Colon vom Hund wurden säurestabile, niedermolekulare Proteaseninhibitoren angereichert. Sie hemmen Trypsin und Chymotrypsin. Der Hemmstoff aus mẹschlichem Material vermag zusätzlich Proteasen aus menschlichen Leukocyten zu hemmen.

\section{Detection of proteinaseinbibitors in the intestinal tract of man and dog}

Low molecular-weight acid stable proteinaseinhibitors are extracted from homogenzied human colon and dog duodenum, jejunum, ileum and colon. Trypsin and chymotrypsin are inhibited by this products. The inhibitor from human material also inhibits proteases from human leucocytes.

Die Sekrete der Schleimhäute der oberen Luftwege des Menschen enthalten erhebliche Mengen eines niedermolekularen polyvalenten Proteaseninhibitors $(1,2)$. Dieser Inhibitor vermag neben Trypsin und Chymotrypsin den Hauptteil der proteolytischen Enzyme aus zerfallenden Leukocyten zu hemmen (3). Diese Eigenschaft erlaubte uns, für diesen Hemmstoff eine physiologische Funktion abzuleiten $(4,5,6)$. Der Hemmstoff konnte auch in isolierten Nasen- und Bronchialschleimhäuten nachgewiesen werden (7). Wir nehmen deshalb an, daß dieser Inhibitor ein spezifisches Produkt der Schleimhäute ist. Möglicherweise wird er durch die Drüsenelemente der Schleimhäute, die Becherzellen, in das Sekret sezerniert. Diese Beobachtungen regten uns an, den Intestinaltrakt des Menschen und des Hundes auf das Vorkommen von niedermolekularen Inhibitoren zu untersuchen, da die Schleimhäute des Intestinaltrakts ebenfalls Becherzellen enthalten.

\footnotetext{
Material und Methoden

Untersuchungsmaterial

An menschlichem Material standen uns nur Operationspräparate von Colonresektionen zur Verfügung. Beim Hund wurden Duodenum, Ileum, Jejunum und Colon getrennt untersucht.

Enzyme: Trypsin (EC 3.4.4.4.) vom Rind, behandelt mit NTosyl-L-phenylalanin-chlormethan Merck; Chymotrypsin (EC 3.4.4.5.) vom Rind, behandelt mit $\mathrm{N}^{\alpha}$-Tosyl-L-lysyl-chlormethan Merck; Plasmin (EC 3.4.4.14.) Novo; Leukocytenproteasen aus menschlichen Leukocyten, Laborpräparat. $0,1 \mathrm{mg}$ unseres Präparates bewirkt unter den bei Untersuchungsmethoden für dieses Enzymgemisch angegebenen Bedingungen eine Spaltung von Hämoglobin, die sich in einet Extinktion von 0,64 der gebildeten Spaltprodukte nach Fätbung mit Reagenz nach FolINCrocaulteau zeigt.
}

Substrate

$\mathrm{N}^{\alpha}$-Benzoyl-D,L-arginin-4-nitroanilid-hydrochlorid Merck; Succinyl-L-phenylalanin-4-nitroanilid, Boehringẹr; Hämoglobin, Serva.

Untersuchungsmethoden

Trypsinbemmung

Die Hemmwirkung der Extrakte gegen Trypsin wurde anhand der Verminderung der Hydrolysegeschwindigkeit von $\mathrm{N}^{\alpha-}$ Benzoyl-D,L-arginin-4-nitroanilid durch Trypsin in Standardansätzen bestimmt. Eine Inhibitormilliunit $(\operatorname{ImU})$ ist in einem Konzentrat vorhanden, wenn die Wirkung einer mU Trypsin aufgehoben wird. Bei reinen Trypsin- und Chymotrypsinpräparaten entspricht etwa ein $\mu \mathrm{g}$ Enzymprotein einer mU Enzym. Definition der Trypsineinheit und experimentelle Einzelheiten siehe 1 . c. (8).

\section{Chymotrypsinbemmung}

Die Hemmwirkung gegen dieses Enzym wurde analog anhand der Verminderung der Spaltung von Succinyl-L-phenylalanin4-nitroanilid ermittelt. Experimentelle Einzelheiten siebe 1. c. (8).

Plasmin

Dieses Enzym spaltet wie Trypsin $\mathrm{N}^{\alpha}$-Benzoyl-D,L-arginin-4nitroanilid. In analogen Ansätzen wie bei Trypsin ist es möglich, die Hemmwirkung der Inhibitorkonzentrate gegen dieses Enzym zu prüfen.

\section{Leukocytenproteasen}

Zur Prüfung auf Hemmwirkung gegen Leukocytenproteasen wurde die Verminderung der Spaltung von Hämoglobin in Gegenwart von Inhibitor untersucht.

Ansätze: Enzym (im vorliegenden Fall $300 \mu \mathrm{g}$; einzusetzende Menge abhängig von der Güte des Proteasenpräparats) plus Inhibitor plus Puffer (Borat $50 \mathrm{mmol} / \mathrm{l}, \mathrm{pH} 7,5$ ) Gesamtvolumen $0,5 \mathrm{ml}, 10 \mathrm{~min}$ vorinkubieren, dann $2,5 \mathrm{ml}$ Hämoglobinlösung ( $20 \mathrm{~g} / \mathrm{I}_{2}$ Harnstoffdenaturiert) im gleichen Puffer (9) zugeben und $2 \mathrm{~h}$ bei $37^{\circ} \mathrm{C}$ inkubieren. Nach Zugabe von $5 \mathrm{ml}$ Trichloressig- 
säure $(50 \mathrm{~g} / 1)$ zentrifugieren, $2,5 \mathrm{ml}$ Uberstand mit $5 \mathrm{ml} 0,5 \mathrm{~mol} / 1$ $\mathrm{NaOH}$ neutralisieren und $1,5 \mathrm{ml}$ Folinteagenz zugcben. Nach Zentrifugieren bei $578 \mathrm{~nm}$ Extinktion gegen Reagenzienleerwert bestimmen. Unter diesen Bedingungen ergibt die ungehemmte Reaktion gegenüber dem Reagenzienleerwert eine Extinktionsdifferenz von 0,20 .

Im vorlicgenden Fall ist es notwendig, einen Leerwert, der nur Inhibitor und Substrat enthält, zusätzlich zu bestimmen. Die Inhibitorpräparate enthalten $g$ stinge Mengen unspezifischer, nicht hemmbarer Proteasen, die eine zu geringe Hemmwirkung der Präparate vortäuschen können. Die Ansätze werden genau so lange inkubiert wie die Hemmansätze. Unmittelbar vor Fällung wird Protease zugesetzt. Die von den Leukocytenproteasen ausgehende Aktivität bzw. die in Gegenwart verschiedener Inhibitormengen verbleibende Restaktivität entspricht der Extinktionsdifferenz der Parallelansätze.

\section{Anreicherung der Inhibitoren}

Die Darmabschnitte werden durch Spülen mit physiol. $\mathrm{NaCl}$ Lösung gereinigt und sofort in Perchlorsäure $(40 \mathrm{~g} / 1)$ homogenisiert. Nach Zentrifugieren wird der Uberstand vorsichtig mit konzentrierter $\mathrm{KOH}$ unter Kühlung bis zu einem $\mathrm{pH}$-Wert von 6 versetzt. Der Uberstand wird vom ausgeschiedenem $\mathrm{KClO}_{4}$ abdekantiert und im Vacuum eingeengt. Es werden stark opaleszierende Lösungen erhalten, mit denen keine optischen $\mathrm{Hemm}$ tests durchführbar sind. Durch Einfrieren und Auftauen ist es möglich, die Trübungsstoffe zur Aggregation zu bringen, sie sind dann abzentrifugierbar. Die weitere Reinigung und Anreicherung erfolgt durch Gelfiltration an Sephadex G 75 in Borat, $50 \mathrm{mmol} / \mathrm{l}$ pH 8,0. Die Inhibitor enthaitenden Fraktionen werden lokalisiert durch Bestimmung der Hemmwirkung entnommener aliquoter Teile gegen Trypsin. Die Hemmstoff enthaltenden Fraktionen werden vereinigt und durch Druckdialyse so weit eingeengt, daß Lösungen mit einem Inhibitorgehalt von $25 \mathrm{ImU} / \mathrm{ml}$ erhalten werden. Die erreichbare spez. Aktivität beträgt $55 \mathrm{ImU} / \mathrm{mg}$ Trockensubstanz. Aus $100 \mathrm{~g}$ Darmgewebe werden im Durchschnitt 135 ImU bei diesem Vorgehen gewonnen.

\section{Ergebnisse}

$\mathrm{Zu}$ Beginn der Untersuchungen wurde der Inhibitorgehalt der einzelnen Darmabschnitte nach Homogenisieren in Perchlorsäure, bezogen auf Frischgewicht und Trypsinhemmung, bestimmt. Wie die Tabelle 1 zeigt, sind die Hemmstoffgehalte sehr niedrig. Dies ist verständlich, da die Isolierung der Mucosa in größeren Mengen problematisch ist und daher das gesamte Darmgewebe für die Untersuchungen verwendet wurde. An die Isolierung größerer Inhibitormengen und an eine Reindarstellung durch Affinitätschromatographie war aus diesen Gründen nicht zu denken. Zudem stellten wir fest, daß die erhaltenen Inhibitorpräparate rasch ihre Wirksamkeit verlieren. Die Ursache dafür dürften geringe Mengen unspezifischer, nicht hemmbarer Proteasen in den Präparaten sein, die zu entfernen bisher nicht gelungen ist.

Tab. 1

Inhibitorgehalt verschiedener Darmregionen

(T. muscularis et mucosa)

\begin{tabular}{llc}
\hline Species & Darmregion & $\begin{array}{c}\text { Inhibitorgehalt } \\
\text { [ImU/g] }\end{array}$ \\
\hline Mensch & Colon & 5,5 \\
Hund & Duodenum & 7,0 \\
Hund & Jejunum & 3,8 \\
Hund & Ileum & 2,0 \\
Hund: & Colon & 7,4 \\
\hline
\end{tabular}

Das Inhibitorkonzentrat aus menschlichem Material vermag Trypsin, Chymotrypsin und leukocytäre Proteasen zu hemmen. Daß die Hemmung nicht durch unspezifische Effekte zustande kommt, zeigen die entsprechenden Hemmkurven. Es ist die durch steigende Mengen Inhibitorkonzentrat erzielbare Hemmung definierter Enzymmengen graphisch dargestellt (Abb. 1 u. 2). Der Hemmtyp ist völlig identisch mit Hemm-

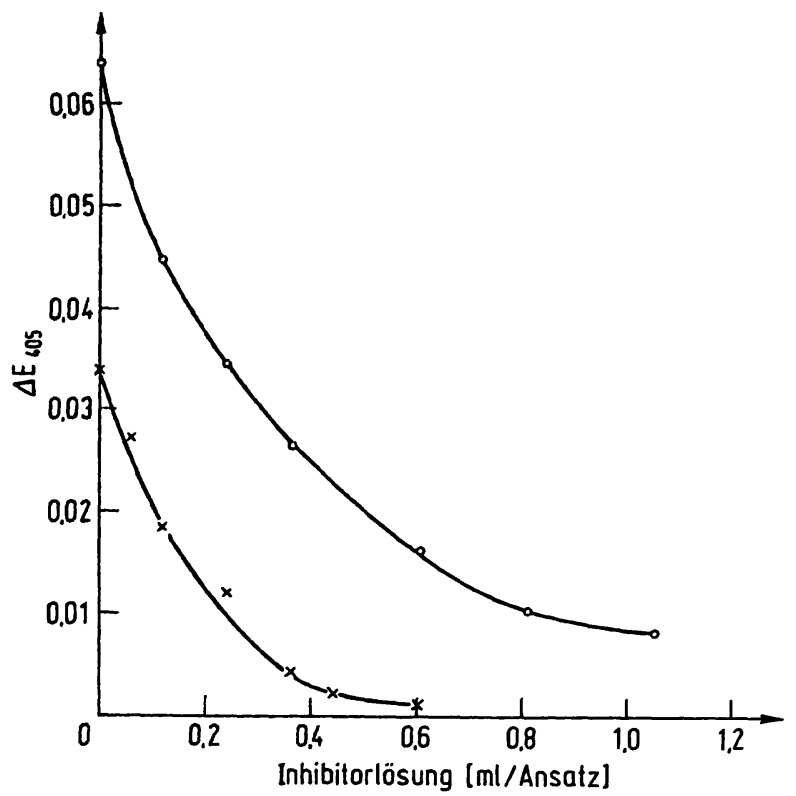

Abb. 1

Hemmwirkung von angereichertem Inhibitor aus menschlichem Colon egen Trypsin $(x-x)$ und Chymotrypsin $(0-O)$ Konstante Enzymmengen, steigende Inhibitorkonzentration pro An-

Trypsin: im Ansatz $10 \mathrm{mU}$ Trypsin, Inhibitorlösung, TriäthanolaminHCl-Puffer $02 \mathrm{~mol} / 1 \mathrm{pH} 7,8$ bis Éndvolumen $2 \mathrm{ml}$. $1 \mathrm{ml}$ Substratlösung, Endkonzentration $73 \mu \mathrm{mol} / 1 . \Delta \mathrm{E}_{105} / \mathrm{min}$ der ungehemmten ReChymotrypsin: im Ansatz $20 \mathrm{mU}$ Chymotrypsin, Inhibitorlösung Puffer wie bei Trypsin. Substrat $1 \mathrm{ml}$, Endkonzentration $0,43 \mathrm{mmol} / \mathrm{l}$. $\Delta E_{40 \text { s } / 30}$ min der ungehemmten Reaktion 0,065

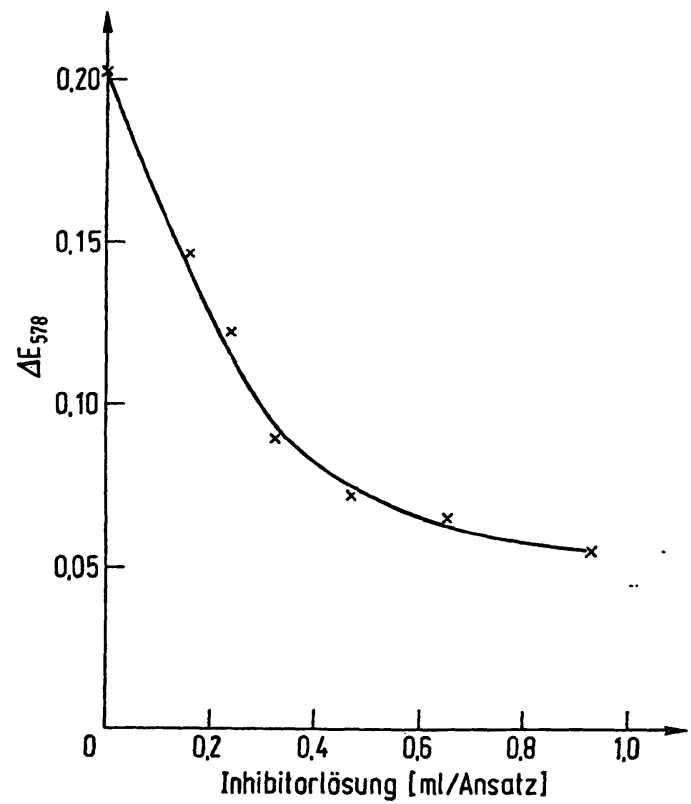

Abb. 2

Hemmwirkung von angereichertem Inhibitor aus menschlichem Colon gegen Leukocytenproteasen

Konstante Enzymmenge ( $300 \mu \mathrm{g})$, steigende Inhibitormenge im Ansatz Ausführung s. Text. Ordinate $\Delta \mathrm{E}_{3}$, nach Umsetzen aliquooter Teile mit Folinreagenz. $\triangle \mathrm{E}$ der ungehemmten Reaktion 0,20 
typen, wie sie mit anderen Inhibitoren erhalten werden $(10,11,1 ; 2)$. Die Trypsinhemmung ist bis zu einem Hemmgrad von $50 \%$ direkt der Inhibitormenge proportional. Bei höheren Hemmgraden macht sich, wie bekannt, die Dissoziation der Enzyminhibitorkomplexe bemerkbar. Ähnlich wie bei Trypsin liegen die Verhältnisse bei der Chymotrypsinhemmung. 50\% Hemmung bei Trypsin (10 $\mathrm{mU}$ Enzym im Ansatz) ist durch etwa $5 \operatorname{ImU}$ (definitionsgemäß) erreichbar, während die gleiche Hemmung bei Chymotrypsin $(20 \mathrm{mU}$ Enzym im Ansatz) durch die etwa doppelte Hemmstoffmenge erreicht wird. Daraus kann abgeleitet werden, daß der Inhibitor jeweils ein Molekül Trypsin oder Chymotryp$\sin z u$ inhibieren vermag. Vorausgesetzt ist hierbei, daß kein Gemisch aus einem monovalenten Trypsin- und einem monovalenten Chymotrypsininhibitor vorliegt. Dies ist jedoch unwahrscheinlich, da die Proportionalität der Hemmwirkung beider Enzyme bei verschiedenen Inhibitorpräparationen konstant bleibt.

Die Proteasen des Cytosols von Leukocyten stellen ein komplexes Gemisch verschiedener Proteasen dar (12, 13). Die Hauptaktivität bei menschlichen Leukocyten entfällt auf ,alkalische" Proteasen, die durch den niedermolekularen Inhibitor der oberen Luftwege (3) und durch $\alpha_{1}$-Antitrypsin des Blutserums (14) hemmbar sind. Die nur partielle Hemmbarkeit der Proteasen des Cytosols wird durch die Anwesenheit nichthemmbarer Proteasen bewirkt (Abb. 2). Plasminhemmung konnte nicht festgestellt werden.

Aus dem Elutionsvolumen der Gelfiltration kann auf ein Molekulargewicht von etwa 10000-12000 geschlossen werden. Es liegt damit in der Größenordnung wie das Molekulargewicht der Sekretinhibitoren (14000). Auf eine Identität mit den Sekretinhibitoren der Luftwege kann aus den vorliegenden Befunden noch nicht geschlossen werden.

Die Inhibitoren aus den verschiedenen Darmabschnitten des Hundes geben sowohl für Trypsin wie für Chymotrypsin identische Hemmkurven (Abb. 3). Die Hemmung von Chymotrypsin ist jedoch wesentlich schwächer wie beim menschlichen Inhibitor. Eine Beziehung

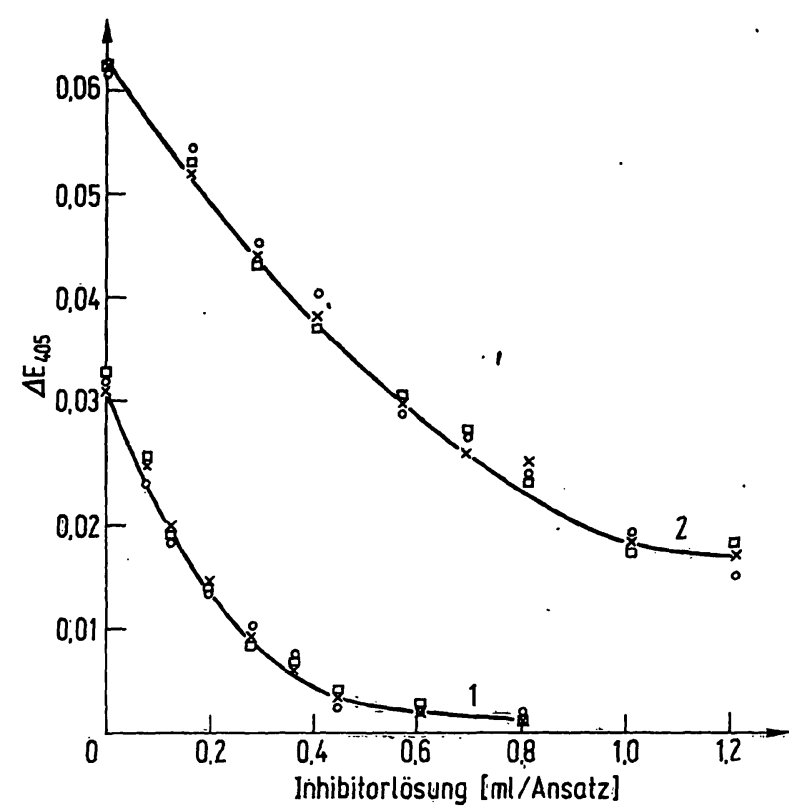

Abb. 3

Hemmwirkung von angereichertem Inhibitor aus dem Intestinaltrakt des Hundes gegen Trypsin (Kurve 1) und Chymotrypsin (Kurve 2). Bedingungen wie Abb. 1 $\times$ Duodenum, $\square$ Ileum, $O$ Colon

auf molarer Basis zwischen beiden Hemmwirkungen kann nicht hergestellt werden. Es wäre auch hier denkbar, daß zwei verschiedene Inhibitoren in verschiedener Konzentration vorliegen. Die konstante Relation der Hemmwirkung gegen beide Enzyme durch Präparate aus verschiedenen Darmabschnitten spricht jedoch gegen diese Interpretation.

Hemmung von Plasmin konnte mit diesem Inhibitor nicht erreicht werden. Ebenso war keine Hemmung der menschlichen leukocytären Proteasen feststellbar.

Vorstellungen über die physiologische Funktion von Proteaseninhibitoren im Intestinaltrakt können vorerst nur auf spekulativer Basis entwickelt werden. Infolgedessen verzichten wir auf eine Diskussion über die Bedeutung der vorgelegten Ergebnisse.

Diese Arbeit wurde mit Mitteln des SFB 51 der Deutschen Forschungsgemeinschaft gefördert.

\section{Literatur}

1. Hochstrasser, K., Haendie, H., Reichert, R. \& Werle, E. (1971), Hoppe-Seyler's Z. Physiol. Chem. 352, 954-958. 2. Hochstrasser, K., Reichert, R., Schwarz, S. \& Werte, E. (1972), Hoppe-Seyler's Z. Physiol. Chem. 353, 221-226. - 3. Hochstrasser, K., Reichert, R., Matzner, M. \& Werie, E. (1972), diese Z. 10, 104-107. - 4. ReICHERT, R., HochstrasSER, K. \& WerLe, E. (1971), Klin. Wochenschr. 49,1234-1236. 5. Reichert, R. \& Hochstrasser, K. (1972), Z. Laryngol. Rhinol. 51, 73-80. - 6. ReIchert, R., HOCHSTRASSER, K. \& Conradi, G. (1972), Pneumonologie 147, 13-20. - 7. ReIchert, R. \& Hochstrasser, K. i. V. - 8. Frutz, H., Trautschold, I. \& Werle, E. (1970), in Methoden der enzymatischen Analyse
(Bergmeyer, H. U., Hrsg.) Aufl. 2 Bd. I, S. 1021-1037, Verlag Chemie, Weinheim/Bergstr. - 9. RIck, W., ebenda Bd. I, S. 975. 10. Fritz, H., Jaumani, E., Meister, R., Pasquay, P., HochSTRAsser, K. \& Fink, E. (1971), in Proc. Int. Res. Conf. on Proteinase-Inhibitors (Fritz, H. \& Tschesche, H., Hrsg.), S. 257-270. - 11. Fritz, H., Schramm, W., Greif, B., Hochstrasser, K., FinK, E. \& Werle, E. (1970), Hoppe=Seyler's Z. Physiol. Chem. 351, 145-150. - 12. JANOFF, A! (1972), Ubersicht in Annual Rev. of Medicine 23, 177-191. - 13. KoptTAR, M., KREgar, I. \& Lebez, D. (1971), Enzymologia 41, 129-139. - 14. LrbermanN, I. \& Gawas, M. A. (1971), J. Lab. Clin. Med. 77, 713-727.
Priv.-Doz. Dr. K. Hochstraßer HNO-Klinik der Universität 8 München 2

Pettenkoferstr. 4a 
fur die kolorimetrische Bestimmung von Lactatdehydrogenase (LDH) mil dem Auto Analyzer@, AA-II@ und SMA@-Verfahren.

Das Lyophilisat ist zur Herstellung der Gebrauchslösung mit Wasser aufzufullen.

$$
\text { Bestellen Sie: Lager-Nr. 940-3 25-ml-Packung, lyophilisiert, \$ 7,75 }
$$

GröBere Mengen auf Anfrage - Wir garanfleren die besten Preise

Versand mit Luftiracht in alle Welt ist im Preis inbegriffen

Für Laboratorien, die die Mischung selbst herstellen wollen, bieten wir das beste NAD und Diaphorase einzeln und zu konkurrenzlosen Preisen an.

Ferner erhällich von Sigme - hergesfellt bei Sigme

\section{MDH/NADH}

(DPNH/MDH; NADH/MDH) Technicon Reference No. 0444

für die UV-Bestimmung von Aspartattransaminase (SGOT) mit dem AA-IIB und SMA@-Verfahren.

Das Lyophilisat ist zur Herstellung der Gebrauchslösung mit Wasser aufzufüllen.

Größere Mengen auf Anfrage

Bestellen Sle: Lager Nr. 936-3 50-ml-Packung, lyophilisiert, 12 Packungen $\$ 37,50$

Versand mit Lufffracht in alle Welt ist im Preis inbegriffen

Wenn Sle thre Mischung selbst herstellen wollen, fragen Sie Sigma nach NADH (DPNH) und Malatdehydrogenase (MDH) höchster Reinheit.

(B) Eingetragene Warenzeichen der Technicon Corporation

Schenken Sle den Gerüchten elnes fehlgeleiteten Konkurrenten, der der einzige Hersteller von Dlaphorase und bestimmter anderer Reagenzien zu sein behauptet, kein Gehör.

Sigma ist der Welt bedeutendster Hersteller fast aller wichtigen Enzyme und Substrate, die für automatisierte und manuelle Verfahren gebraucht werden. Alle oben aufgefürten Reagenzien (Diaphorase, NAD, NADH und Malaidehydrogenase (MDH)) werden bel Sigma selbst hergestellt.

Wir garantieren höchste Qualität bei niedrigsten Preisen!

Es ist ein Vergnügen, Geschafte mit Sigma zu machen

Besfellen Sle direkt - R-Gespräch von überall her in der Welt Tagsüber von Haus zu Haus, 314-771-5750. Nachts von Person zu Person, Dan Broida, 314-092-6418 TWX (Fernschreiber) Tag und Nacht: 910-761-0593

Sigma-Reagenzien sind in der ganzen Welf durch den Fachhandel oder direkt aus St. Louis beziehbar. Telegramme: SIGMACHEM, St. Louis, Missouri

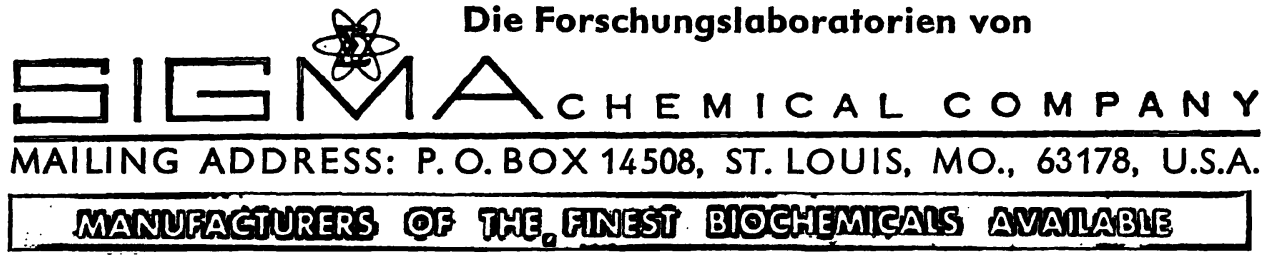

SIGMA LONDON Chem. Co. Vertreten durch

Telephone: 01-736-5823 (Reverse Charges)
SIGMA ISRAEL Chem. Co. Ltd. . 28 Kaf-Gimel St, Givataim, Israel Telephone: (03) 760654 (Reverse Charges)

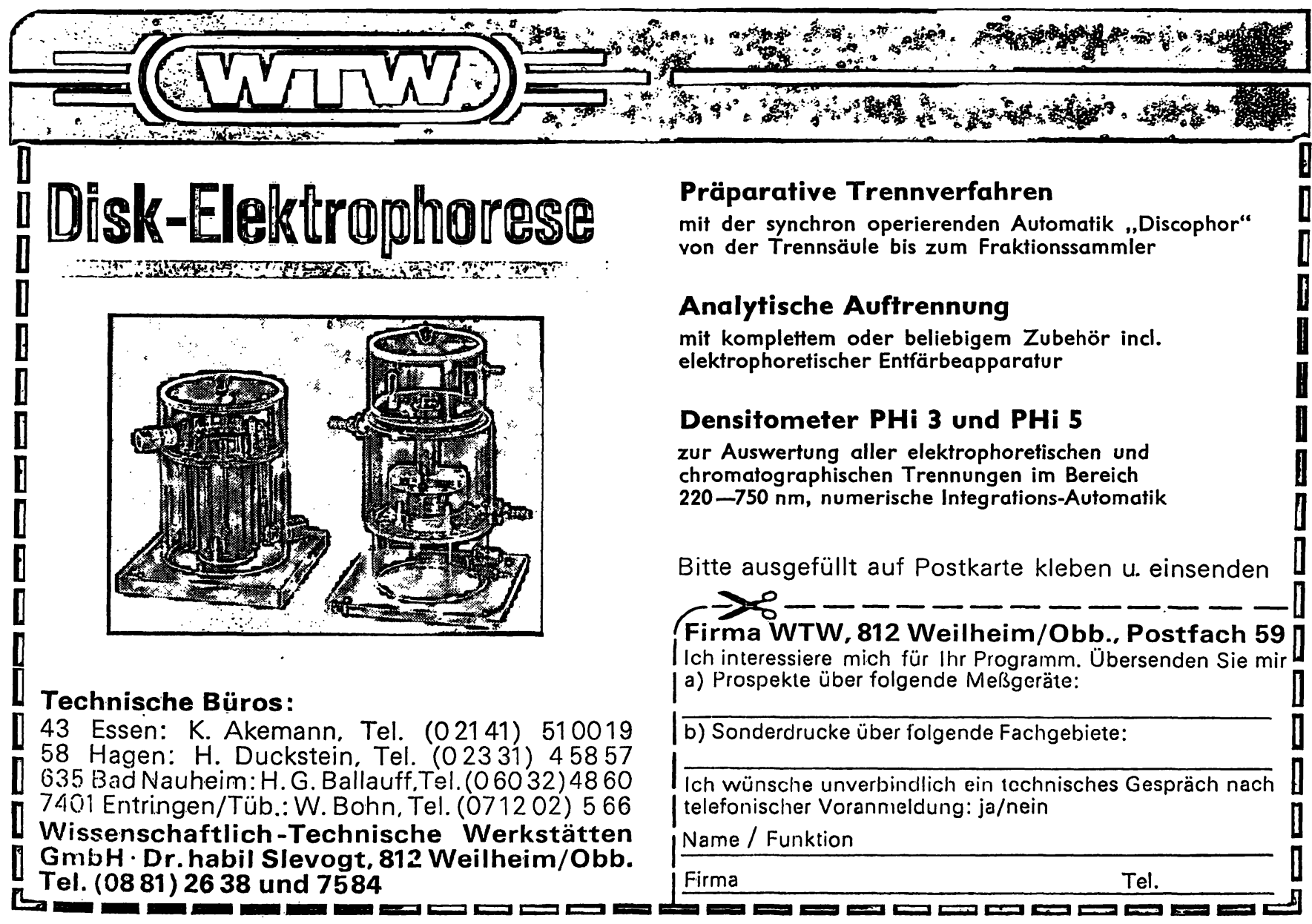




\section{Elugieren Sie}

quantitative TrennzOMEn

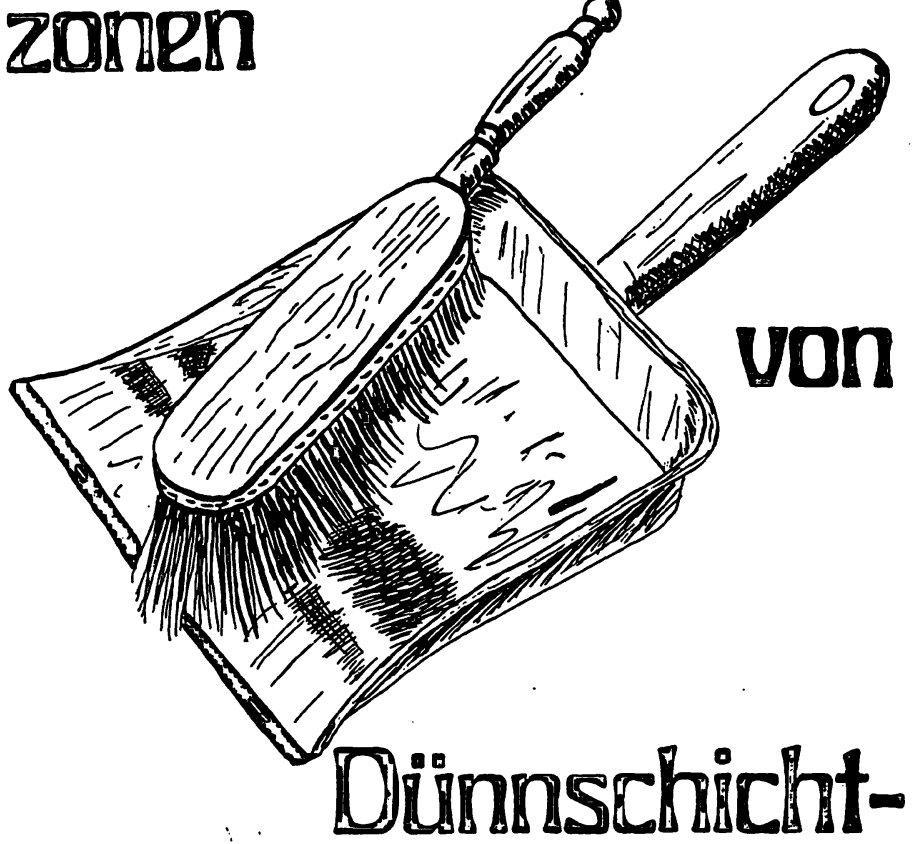
Chromatogrammen?

Dann haben wir eine gute Nachricht für Sie.

Sie brauchen das nicht mehr zu tun nach der Methode: abkratzen

auffangen, extrahieren

filtrieren oder zentrifugieren

dekantieren, auffüllen

pipetțieren, messen

sondern ab jetzt können Sie

\section{direkt von der Platte}

in die Küvette eluieren,

messen,

und zwar mit sechs Trennzonen gleichzeitig und automatisch. Mit dem CAMAG ELUCHROM.

Eingesparte Arbeitsgänge sind eingesparte Fehlerquellen. Mitunter ist man auch froh über eingesparte Arbeitszeit.

Möchten Sie über das CAMAG ELUCHROM näheres erfahren? Schreiben Sie uns.

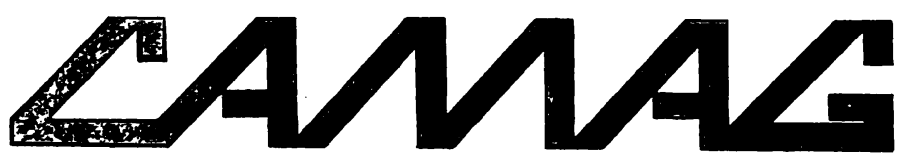

Führend in Dünnschicht-Chromatographie Dünnschicht-Elektrophorese Hochspannungs-Elektrophorese

4132 Muttenz/Schweiz

Homburgerstrasse 24

Tel. (061) 531430
1000 Berlin 41

Bismarckstrasse 27-29

Tel. (0311) 7915091
Neuerșcheinung

\section{Fuchs - Freiwald}

\section{Allgemeine und anorganische Chemie}

Einführung in die Grundlagen für Mediziner, Naturwissenschaftler und Chemie-Nebenfächler

Von Prof. Dr. JOACHIM FUCHS und Dipl.-Chem. WILFRIED FREIWALD, Instituf für Anorganische Chemie der Freien Universität Berlin

Groß-Okłav. VI, 174 Seiten. 1972. Kartoniert DM 12,80 ISBN 3110042436

Der "Lerntext" soll in kürzester, aber dennoch verständlicher Form die wichtigsten Grundlagen der anorganischen und allgemeinen Chemie-darstellen. Dabei ist der ,Lernstoff" auf ein Minimum beschränkt, das Hauptgewicht wird auf ein Vermitteln allgemeiner Gesetzmäßigkeiten und Zusammenhänge gelegt.

Nach Kapiteln über Atombau und Periodensysfem wird die Chemie der Elemente, geordnet nach den Gruppen des Periodensystems, bèsprochen, wobei an prägnanten Beispielen Gebiete aus der allgemeinen und physikalischen Chemie (Reaktionskinetik, Thermodynamik, Elektrochemie) in einfacher Form behandelt werden. Der Aufbau ist so gewählt, daß die Eigenschaften und Reaktionen der Stoffe mit Hilfe der jeweils bereits erklärten theoretischen Grundlagen anschaulich verständlich sind. Schon nach einem einmaligen intensiven Durcharbeiten des Stoffes wird ein hoher Lerneffekt erzielt.

Das Buch ist speziell für Studenten mit Chemie als Nebenfach (Mediziner, Biologen, Physiker u. a.) gedacht. Es sollte aber auch für Studenten der Chemie als Hauptfach und für Chemotechniker an Ingenieurakademien und Fachhochschulen in den Anfangssemestern von Nutzen sein, um zu erkennen, welche Gebiete der Chemie, die in ausführlichen und umfangreichen Lehrbüchern behandelt werden, von besonderer Wichtigkeit sind. 